\title{
EFECTO DE PRETRATAMIENTOS EN SEMILLAS DE ENTEROLOBIUM CONTORTISILIQUUM (FABACEAE) DE LA SELVA PEDEMONTANA ARGENTINA
}

\author{
EVANGELINA C. LOZANO ${ }^{1}$, MARIA A. ZAPATER, CECILIA MAMANI, CAROLINA B. \\ FLORES, MIRIAM N. GIL y SILVIA S. SÜHRING
}

\begin{abstract}
Summary: The effect of pretreatments in Enterolobium contortisiliquum seeds (Fabaceae) from Selva Pedemontana of Argentina. Enterolobium contortisiliquum (Vell.) Morong is a tree species that lives in the Selva Pedemontana of Argentina, that is extensively exploited for ample crop. Its seeds typically exhibit low germination percentage due to the high level of dormancy. Five different methods to overcome dormancy were evaluated on seeds collected for five populations along a latitudinal and altitudinal gradient in the Selva Pedemontana. All seeds were germinated at $24^{\circ} \mathrm{C}$ and $12 \mathrm{~h}$ photoperiod. Total germinated seeds and mean time to germinate was recorded. Mechanical scarification and chemical scarification with sulfuric acid for 30 to 50 minutes were highly efficient in promoting germination.
\end{abstract}

Key words: Dormancy, germination, seeds, Selva Pedemontana.

\begin{abstract}
Resumen: Enterolobium contortisiliquum (Vell.) Morong es una especie arbórea que habita en la Selva Pedemontana de Argentina, área muy afectada por cultivos extensivos, cuyas semillas típicamente presentan bajo poder germinativo debido a un elevado grado de dormición. Se aplicaron cinco métodos diferentes para ruptura de dormición a semillas recolectadas en cinco poblaciones en un gradiente de la Selva Pedemontana. La germinación se desarrolló a $24{ }^{\circ} \mathrm{C}$ y con fotoperiodo de 12 horas. Se registró el total de semillas germinadas y la velocidad de germinación. La escarificación mecánica y la escarificación química con ácido sulfúrico por 30 y 50 minutos fueron los tratamientos altamente eficientes para promover la germinación.
\end{abstract}

Palabras clave: Dormición, germinación, semillas, Selva Pedemontana.

\section{INTRODUCCIÓN}

Enterolobium Mart. (Ingeae, Mimosoideae, Fabaceae), reúne 11 especies arbóreas de alto valor maderable, que se distribuyen desde México y Centroamérica a Sudamérica, con una mayor concentración de especies en Argentina, Bolivia, Brasil, Paraguay y Uruguay (Lewis \& Rico Arce, 2005). Para Argentina, Hoc (2005) cita una sola especie para el género, E. contortisiliquum (Vell.) Morong, denominada "pacará", "timbó" u "oreja de negro" (Fig.1), con hábitat en las Selvas de Transición de la Provincia Biogeográfica de las

\footnotetext{
1 Facultad de Ciencias Naturales, Universidad Nacional de Salta, Av. Bolivia 5150, 4400 Salta, Argentina; aliciazapater@yahoo.com.ar
}

Yungas (PB de las Yungas), a 120-550 m s.m., en los bosques de timbó de la PB Chaqueña y en las Selvas Marginales (PB Paranaense).

En el noroeste de Argentina, E. contortisiliquum es codominante en la comunidad de la Selva de Tipa y Pacará (Cabrera, 1994), característica del sector sur de la Selva Pedemontana de la PB de las Yungas, en un área actualmente muy modificada y reducida por expansión de la frontera agropecuaria. Las poblaciones remanentes muestran individuos de buen porte, semejantes en morfología y arquitectura, aislados en bordes de rutas y caminos. Además, la especie se encuentra con menor dominancia en el sector norte de las Yungas y en el Chaco serrano húmedo.

E. contortisliquum tiene valor comercial por su madera liviana, que puede sustituir en determinados usos al "cedro salteño" (Cedrella balansae DC.). Se 

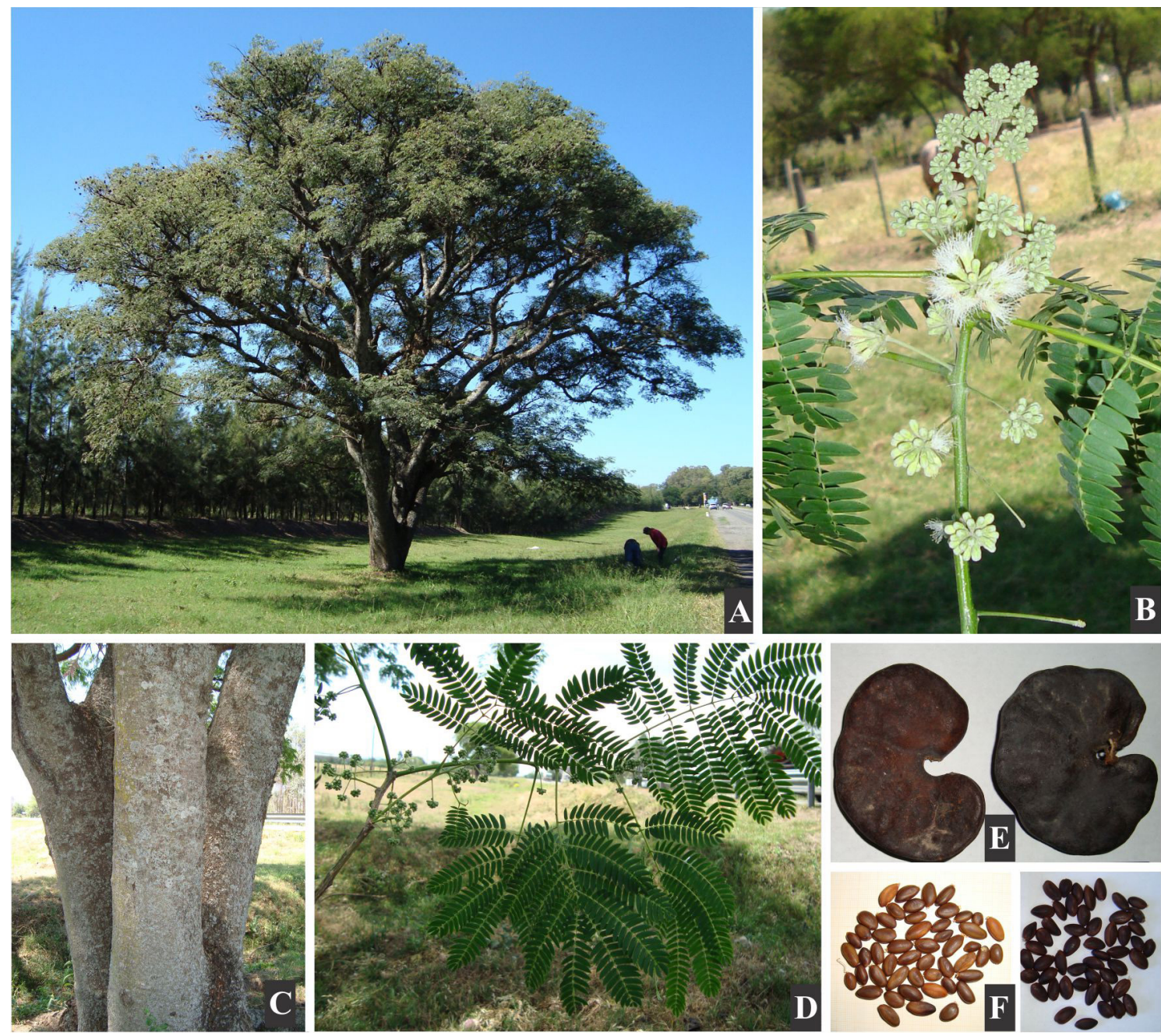

Fig. 1. Enterolobium contortisiliquum. A: Vista de un árbol de la población de Metán (Salta). B: Inflorescencia. C: Detalle de la corteza. D: Hojas. E: Frutos. F: Semillas. De Zapater et al. 3088, 3091, 3094 (MCNS). Fotografías E. C. Lozano.

emplea para elaborar envases, colmenas, persianas, interiores y exteriores de muebles (Tortorelli, 2009); también como árbol ornamental. Por otra parte, E. contortisiliquum es una especie pionera con rápido crecimiento, que ha sido recomendada para la recomposición de áreas degradadas (Barth et al., 2008; Alexandre et al., 2009). Por esto constituye un óptimo material para cultivo en la Selva Pedemontana de las Yungas con el objeto de restaurar sitios degradados, para enriquecer cortinas forestales en los campos de cultivos extensivos y establecer sistemas de enriquecimiento del bosque nativo.
Las semillas de Enterolobium, al igual que en otros géneros de Fabaceae, se caracterizan por poseer una cubierta impermeable constituida por una capa con células en empalizada formada por macroesclereidas (células de Malpighi), impregnadas con sustancias hidrofóbicas (Baskin \& Baskin, 2014), lo que induce dormición física. En Fabaceae el ingreso del agua en la semilla ocurre en una región anatómica especializada (lente), que en condiciones favorables actúa como una válvula higroscópica permitiendo la imbibición de las semillas (Baskin et al., 2000; Baskin \& Baskin, 2014). 
Por la impermeabilidad del tegumento, la germinación de las semillas de E. contortisiliquum es muy irregular (Eira et al., 1993; Lima et al., 1997, Ramírez et al., 2012), lo que caracteriza a la mayoría de las especies pioneras o predominantes de etapas serales secundarias (Hopkins \& Grahan, 1987), como estrategia de sobrevivencia en el estadio inicial de la sucesión ecológica (Alexandre et al., 2009).

Diversos métodos artificiales han sido descriptos y utilizados para romper la dormición física de las semillas, tales como la escarificación mecánica, la química con ácido sulfúrico y la térmica con calor húmedo (Baskin \& Baskin, 2014). La aplicación de ácido sulfúrico actuaría desagregando en porciones la cubierta seminal, produciéndose una rápida imbibición; en tanto que los tratamientos de calor húmedo provocarían la apertura del lente (ruptura) y una imbibición lenta y gradual, similar al proceso en la naturaleza (Li et al., 1999; Baskin \& Baskin, 2014; Galíndez et al., 2015). La aplicación de cualquiera de estos métodos requiere el ajuste del tiempo en que las semillas son sometidas a dichos tratamientos para evitar el daño o la muerte del embrión (Baskin \& Baskin, 2014; Galíndez et al., 2015).

Numerosas experiencias realizadas con las semillas de E. contortisiliquum, mostraron que la efectividad de diferentes pretratamientos es variable, lo que es característico de especies con amplia distribución geográfica, que tienen respuestas diferenciales por efecto de adaptación al origen (Eira et al., 1993). Capelanes (1991) obtuvo $100 \%$ de germinación después de $72 \mathrm{~h}$ de inmersión en agua; Eira et al. (1993), utilizando lotes de semillas de ambientes diferentes, concluyeron que el tratamiento con ácido sulfúrico era el más efectivo por promover mayor uniformidad y velocidad de germinación, aunque obtuvieron un $90 \%$ de germinación en dos de los lotes de semillas tratadas con agua caliente. Malavasi y Mattos Malavasi (2004), reportaron que los tratamientos más eficientes fueron la escarificación mecánica y la química con ácido de 30 a 80 minutos, aconsejando la primera para la producción en vivero por razones de practicidad. Una experiencia realizada en Sevilla (Rossini Oliva et al., 2006), con varias plantas cultivadas, dio como resultado que las semillas de E. contortisiliquum germinaron el $100 \%$ y con la mejor velocidad de germinación sin pretratamiento. Esto muestra que el efecto de los tratamientos pregerminativos puede ser diferente entre las poblaciones debido a variaciones genético-ambientales (Eira et al., 1993; Navarro et al., 2002).

Según Bewley y Black (1982), el peso de las semillas es uno de los factores determinantes de la dormición física, porque a medida que el peso disminuye los tegumentos se tornan progresivamente duros e impermeables. Los experimentos de Borges et al. (1980) y Eira et al. (1993) con semillas de diferentes poblaciones de E. contortisiliquum, mostraron que las semillas de inferior peso, colectadas en sitios con clima más seco, tuvieron mayor porcentaje de semillas durmientes sin tratamiento pregerminativo. Algunos autores sugieren, que en condiciones naturales, la exposición de semillas a altas temperaturas o a temperaturas fluctuantes, estimularían la salida de dormición (Galindez et al., 2015).

La semilla es un material barato, práctico y el usualmente empleado para propagar especies, que se debe obtener de ejemplares bien conformados, sanos, a los que se considera árboles deseables (semilleros). En el caso de E. contortisiliquum la obtención de semillas sanas es difícil; por un lado, la extracción de las semillas del fruto se dificulta debido a la dureza del epicarpio coriáceo, la semi-carnosidad del mesocarpio y a que las semillas se encuentran separadas en artejos del endocarpio cartilagíneo. Por otro lado, los frutos presentan un porcentaje de entre 15 y $38 \%$ de semillas depredadas, como fue reportado por Morandini y de Viana (2009) para tres poblaciones de las provincias de Salta y Jujuy. Además, es necesaria la escarificación del duro tegumento de las semillas para lograr una germinación pareja, a los fines de hacer un uso óptimo de las mismas. Bajo este esquema se plantea como objetivo del presente estudio evaluar la eficiencia de los métodos para inducir la ruptura de la dormición en las semillas provenientes de poblaciones distribuidas en un gradiente latitudinal y altitudinal de la Selva Pedemontana de Argentina. Además se analiza la variabilidad en el tamaño de los frutos y semillas y el número de semillas sanas por fruto de estas poblaciones. 


\section{Material y Método}

El área de estudio se sitúa en el piedemonte agrícola de las Yungas en un gradiente latitudinal $\left(26^{\circ} 24^{\prime}-23^{\circ} 15^{\prime} \mathrm{S}\right)$ y altitudinal (809-319 m s.m.), desde el norte de Tucumán hasta el norte de Salta (Fig. 2). Esta área presenta además un gradiente térmico (temperaturas medias anuales de 18,3$21,2^{\circ} \mathrm{C}$ ) y de precipitaciones (medias anuales de 421-902 mm) (Bianchi \& Yañez, 1992; Tabla 1). Se colectaron frutos de cinco poblaciones situadas en Choromoro (Depto. Trancas, Tucumán), General Güemes (Depto. Gral. Güemes, Salta), Hipólito Irigoyen (Depto. Orán, Salta), Metán (Depto. Metán, Salta) y Yuto (Depto. Ledesma, Jujuy) (Fig. 2). En las poblaciones de Güemes y Choromoro, ubicadas hacia el sur del gradiente, se registran menores temperaturas y precipitaciones medias anuales (régimen semiárido), mientras que en la de Metán se registran precipitaciones elevadas a pesar de su ubicación latitudinal intermedia, debido a la proximidad y posición E respecto a la Serranía de Metán (Tabla 1). En las poblaciones de Yuto e Hipólito Irigoyen las precipitaciones y temperaturas medias anuales son más elevadas (Tabla 1).

En cada sitio se seleccionaron al azar 50 frutos, de por lo menos tres individuos de buen porte y sanidad, los que fueron medidos y pesados. Se extrajeron las semillas para registrar el número de semillas por fruto, de las que se seleccionaron al azar 50 semillas sanas para registrar el peso y las dimensiones. Los pesos se determinaron con balanza electrónica. Se realizó un ANOVA para evaluar si el tamaño de frutos y semillas presentan variabilidad espacial.

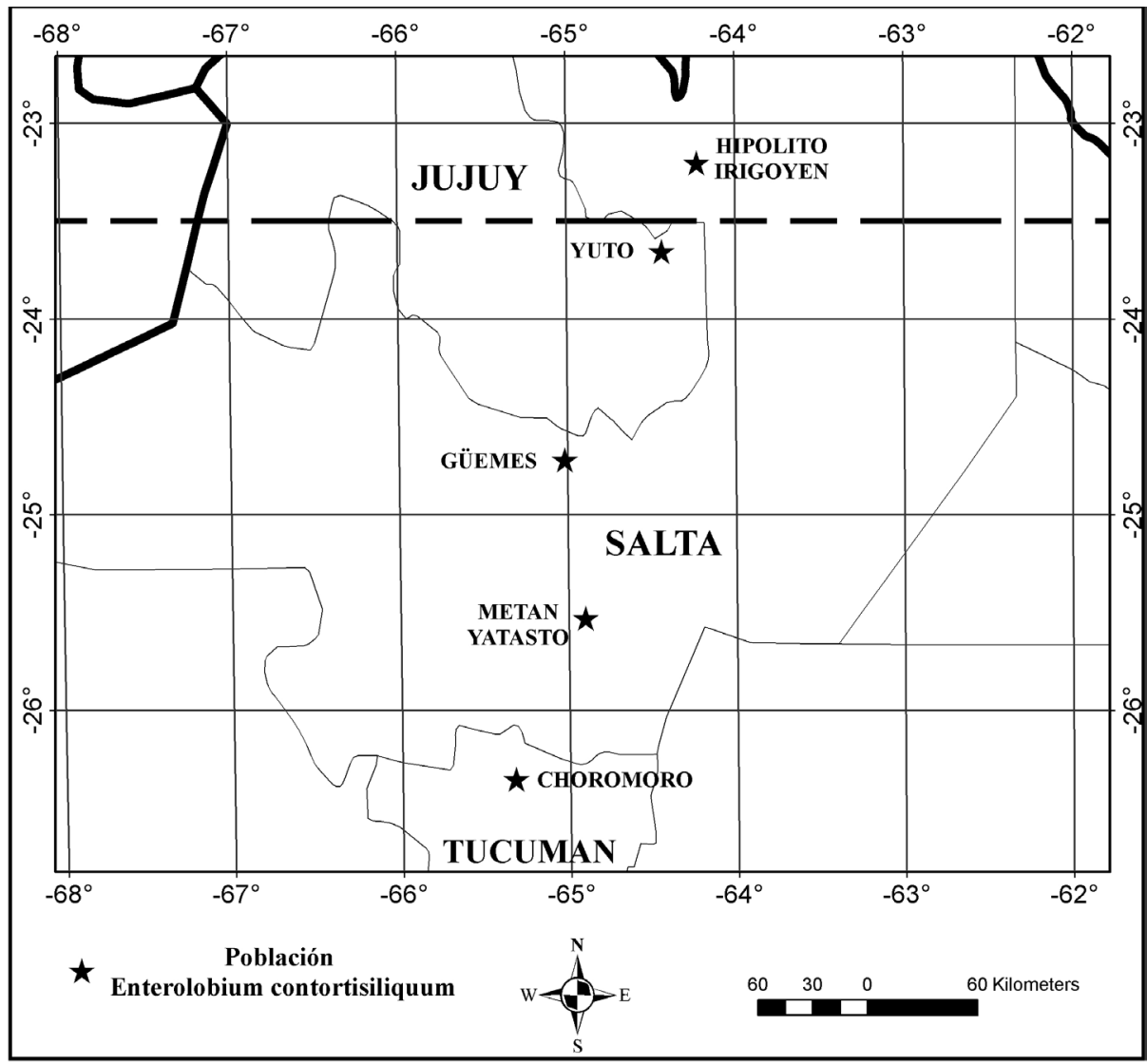

Fig. 2. Distribución geográfica de las poblaciones de Enterolobium contortisiliquum en el área de estudio. 
Tabla 1. Caracterización de variables ambientales y geográficas de las poblaciones de Enterolobium contortisiliquum estudiadas. Altitud en m s.m. PMA: Precipitación media anual en mm. TMA: temperatura media anual en ${ }^{\circ} \mathrm{C}$.

\begin{tabular}{|cccccc|}
\hline Sitio & Latitud S & Longitud W & Altitud & PAM & TMA \\
\hline Choromoro & $26^{\circ} 24^{\prime}$ & $65^{\circ} 19^{\prime}$ & 809 & 421 & 18.6 \\
Güemes & $24^{\circ} 46^{\prime}$ & $65^{\circ} 02^{\prime}$ & 718 & 536 & 19.7 \\
Hipólito Irigoyen & $23^{\circ} 15^{\prime}$ & $64^{\circ} 15^{\prime}$ & 319 & 696 & 21.2 \\
Metán & $25^{\circ} 35^{\prime}$ & $64^{\circ} 57^{\prime}$ & 796 & 902 & 18.3 \\
Yuto & $23^{\circ} 38^{\prime}$ & $64^{\circ} 28^{\prime}$ & 349 & 863 & 20.7 \\
\hline
\end{tabular}

Se realizaron ensayos de germinación para establecer cuál es la mejor metodología de ruptura de dormición en laboratorio, con semillas que previamente fueron inmersas en solución de hipoclorito de sodio al $50 \%$ durante 1 minuto para su desinfección. Los tratamientos aplicados fueron: 1) control (sin ningún tratamiento); 2) escarificación mecánica con lija de agua $\mathrm{n}^{\circ} 120 ; 3$ ) imbibición en agua natural durante $72 \mathrm{~h}$; 4) imbibición en agua caliente a $80^{\circ} \mathrm{C}$ inicial, enfriamiento gradual por $36 \mathrm{~h}$; 5) escarificación química con $\mathrm{H}_{2} \mathrm{SO}_{4}$ al $98 \%$ durante 30 minutos; y 6) escarificación química con $\mathrm{H}_{2} \mathrm{SO}_{4}$ al $98 \%$ durante 50 minutos. Las semillas tratadas con ácido fueron inmediatamente lavadas con agua destilada. Las semillas se dispusieron en cajas de Petri con sustrato de agar (4 repeticiones de 10 semillas por tratamiento), en una cámara de germinación a temperatura constante de $24{ }^{\circ} \mathrm{C}$ y fotoperiodo de 12 horas.

El número de semillas germinadas fue registrado diariamente en el mismo horario hasta completar 30 días, adoptándose como criterio de germinación la sola aparición de la radícula. Se calculó la proporción total de semillas germinadas (p) y la velocidad de germinación (MTG) (Pritchard \& Miller, 1995), al final del experimento. Estas variables de repuesta se analizaron usando un ANOVA, y se utilizó el test DGC para localizar las diferencias cuando el ANOVA resultó significativo. Todos los análisis estadísticos se realizaron con el programa InfoStat (Di Rienzo et al., 2014).

\section{Resultados y Discusión}

Los frutos y semillas de E. contortisiliquum presentan variación espacial, todas las variables métricas registradas presentaron diferencias significativas entre poblaciones $(\mathrm{P}<0,05$; Tablas 2 y 3). Los frutos más pequeños (medias de 12,71$13,12 \mathrm{~g}$ ) correspondieron a las poblaciones situadas en las áreas más áridas del gradiente (Choromoro y Güemes), mientras que los más grandes (más de $21 \mathrm{~g}$ ), se colectaron en las poblaciones con un

Tabla 2. Tamaño (media) de los frutos de Enterolobium contortisiliquum para cada población estudiada.

\begin{tabular}{|cccccc|}
\hline FRUTOS & $\begin{array}{c}\text { Longitud } \\
(\mathbf{c m})\end{array}$ & $\begin{array}{c}\text { Latitud } \\
(\mathbf{c m})\end{array}$ & $\begin{array}{c}\text { Espesor } \\
(\mathbf{m m})\end{array}$ & $\begin{array}{c}\text { Peso } \\
(\mathbf{g r})\end{array}$ & N $^{\mathrm{O}}$ Semillas \\
\hline Choromoro & $20,45^{\mathrm{C}}$ & $2,98^{\mathrm{D}}$ & $11,56^{\mathrm{B}}$ & $13,12^{\mathrm{C}}$ & $14,66^{\mathrm{B}}$ \\
Güemes & $22,1^{\mathrm{B}}$ & $3,35^{\mathrm{C}}$ & $9,08^{\mathrm{D}}$ & $12,71^{\mathrm{C}}$ & $17,8^{\mathrm{A}}$ \\
Hipólito Irigoyen & $24,1^{\mathrm{A}}$ & $3,9^{\mathrm{C}}$ & $10,92^{\mathrm{C}}$ & $21,73^{\mathrm{A}}$ & $13,8^{\mathrm{B}}$ \\
Metán & $23,4^{\mathrm{A}}$ & $3,64^{\mathrm{B}}$ & $11,16^{\mathrm{C}}$ & $16,56^{\mathrm{B}}$ & $14,98^{\mathrm{B}}$ \\
Yuto & $23,4^{\mathrm{A}}$ & $3,66^{\mathrm{B}}$ & $13,04^{\mathrm{A}}$ & $21,96^{\mathrm{A}}$ & $18,58^{\mathrm{A}}$ \\
\hline
\end{tabular}


Tabla 3. Tamaño (media) de las semillas de Enterolobium contortisiliquum para cada población estudiada.

\begin{tabular}{|ccccc|}
\hline SEMILLAS & $\begin{array}{c}\text { Longitud } \\
(\mathbf{c m})\end{array}$ & $\begin{array}{c}\text { Latitud } \\
(\mathbf{c m})\end{array}$ & $\begin{array}{c}\text { Altura } \\
(\mathbf{m m})\end{array}$ & $\begin{array}{c}\text { Peso } \\
(\mathbf{g r})\end{array}$ \\
\hline Choromoro & $1,38^{\mathrm{A}}$ & $0,72^{\mathrm{B}}$ & $5,36^{\mathrm{A}}$ & $0,34^{\mathrm{A}}$ \\
Güemes & $1,06^{\mathrm{C}}$ & $0,55^{\mathrm{B}}$ & $4,54^{\mathrm{C}}$ & $0,22^{\mathrm{C}}$ \\
Hipólito Irigoyen & $1,24^{\mathrm{B}}$ & $0,85^{\mathrm{A}}$ & $4,81^{\mathrm{B}}$ & $0,28^{\mathrm{B}}$ \\
Metán & $1,21^{\mathrm{B}}$ & $0,68^{\mathrm{B}}$ & $4,95^{\mathrm{B}}$ & $0,28^{\mathrm{B}}$ \\
Yuto & $0,95^{\mathrm{D}}$ & $0,58^{\mathrm{B}}$ & $5,01^{\mathrm{B}}$ & $0,27^{\mathrm{B}}$ \\
\hline
\end{tabular}

régimen de precipitaciones mayor a $600 \mathrm{~mm}$ anuales (Hipólito Irigoyen y Yuto). Se registró una correlación positiva significativa $(\mathrm{P}<0.001)$ entre el tamaño del fruto y la cantidad de semillas que contiene, aunque esta asociación no fue muy fuerte en algunas poblaciones (por ejemplo, la relación peso vs. número de semillas fue $r=0.56$ y 0.51 para Güemes y Yuto respectivamente) (Tabla 2). El porcentaje promedio de semillas sanas por fruto de la población de Metán resultó bajo (38 \%) y la depredación alta (62\%); en tanto que en las otras poblaciones el porcentaje de sanas fue elevado, de 70-75\%, con baja depredación (25-30 $\%$ ). Estos resultados muestran que la depredación puede ser mayor a lo citado por Morandini \& de Viana (2009) y variable según las zonas, posiblemente relacionada con el tipo de actividades productivas agropecuarias que en ellas se desarrollan. Las semillas más pequeñas fueron las provenientes de Güemes $(0,22 \pm 0,02 \mathrm{~g})$ con condición semiárida; sin embargo las semillas de Choromoro, también del semiárido, registraron un elevado tamaño $(0,34 \pm 0,04 \mathrm{~g})$ superior a los sitios más húmedos del gradiente (Tabla 4). Es decir, que la variación en el tamaño de las semillas no se correspondería con las condiciones climáticas de los sitios. El porcentaje del peso de semillas respecto del total del fruto (Tabla 4), calculado a partir de los valores promedio para cada población, muestra una aparente relación inversamente proporcional con las precipitaciones y la temperatura. Si bien no se pudo evaluar estadísticamente, el peso total por semillas, que podría corresponder a mayor tamaño o cantidad de semillas fue elevado con respecto al peso del pericarpio del fruto en las áreas con menores precipitaciones y temperaturas medias (Tablas 1 y 4).

La proporción media de semillas germinadas resultó significativamente diferente entre pretratamientos $(\mathrm{F}=$ 230,82; $\mathrm{P}<0,0001)$, así como entre sitios $(\mathrm{F}=4,08$; $\mathrm{P}=0,0044)$. Además estos factores interactúan entre sí $(\mathrm{F}=3,03 ; \mathrm{P}=0,0002)$, es decir, los tratamientos no tienen el mismo efecto en todos los sitios. En todos los sitios los tratamientos con ácido sulfúrico y lijado tuvieron significativamente mayor proporción media de semillas germinadas $(0,90-1,00)$ (Tabla 5). Sólo para Yuto la proporción media de semillas germinadas para el tratamiento agua a $80^{\circ} \mathrm{C}$ fue muy baja y similar a la de agua fría y control. Las semillas sin pretratamiento tuvieron una proporción media muy baja de germinación para la mayoría de los sitios (entre $0-0,13$ ), siendo cero para las colectadas en Güemes, y no se diferenciaron de las proporciones medias obtenidas para el tratamiento con agua fría $(0,03-0,08)$ (Tabla 5).

De acuerdo al ANOVA existen diferencias en el promedio de velocidad de germinación entre las combinaciones sitio-tratamiento $(\mathrm{F}=439,32 ; \mathrm{P}<$ 0,0001) (Fig. 3). La prueba de comparación de medias DGC, mostró que los valores de MTG (Tabla 6),

Tabla 4. Peso (media \pm desviación estándar) de frutos, semillas, número de semillas y relaciones peso semillas y fruto en las poblaciones estudiadas.

\begin{tabular}{|ccccccc|}
\hline Poblaciones & $\begin{array}{c}\text { Peso de } \\
\text { frutos }(\mathbf{g})\end{array}$ & $\begin{array}{c}\text { Peso de } \\
\text { semillas (g) }\end{array}$ & $\begin{array}{c}\mathbf{N}^{\circ} \text { de semillas } \\
\text { totales }\end{array}$ & $\begin{array}{c}\text { \% semillas Peso total de Peso de semillas/ } \\
\text { sanas /fruto semillas (g) }\end{array}$ & $\begin{array}{c}\text { peso fruto (\%) } \\
\text { pesto }\end{array}$ \\
Choromoro & $13,12 \pm 3,02$ & $0,34 \pm 0,04$ & $14,66 \pm 3,19$ & 74,76 & 4,98 & 37,96 \\
Güemes & $12,71 \pm 1,83$ & $0,22 \pm 0,02$ & $17,8 \pm 2,28$ & 70,45 & 3,9 & 30,68 \\
Hipólito Irigoyen & $21,73 \pm 5,5$ & $0,28 \pm 0,041$ & $13,80 \pm 3,21$ & 70,58 & 3,86 & 17,76 \\
Metán & $16,56 \pm 4,4$ & $0,28 \pm 0,07$ & $14,98 \pm 3,86$ & 38 & 4,19 & 25,3 \\
\hline Yuto & $21,96 \pm 4,8$ & $0,27 \pm 0,04$ & $18,58 \pm 2,35$ & 72,22 & 5,02 & 22,86 \\
\hline
\end{tabular}


Tabla 5. Proporción de semillas germinadas (media \pm error estándar) para cada tratamiento de escarificación y sitio de colecta de semillas de Enterolobium contortisiliquum. Letras diferentes indican diferencias significativas entre las medias de acuerdo a la prueba de comparaciones múltiples de medias DGC.

\begin{tabular}{|cccccc|}
\hline Tratamiento & & \multicolumn{3}{c|}{ Poblaciones } & Yuto \\
\hline Testigo & $10 \pm 10^{\mathrm{D}}$ & $0 \pm 0^{\mathrm{D}}$ & $5 \pm 3^{\mathrm{D}}$ & $13 \pm 9^{\mathrm{D}}$ & $5 \pm 5^{\mathrm{D}}$ \\
Lijado & $100 \pm 0^{\mathrm{A}}$ & $93 \pm 0^{\mathrm{A}}$ & $98 \pm 15^{\mathrm{A}}$ & $100 \pm 0^{\mathrm{A}}$ & $100 \pm 0^{\mathrm{A}}$ \\
Agua fría & $3 \pm 3^{\mathrm{D}}$ & $3 \pm 3^{\mathrm{D}}$ & $3 \pm 3^{\mathrm{D}}$ & $8 \pm 3^{\mathrm{D}}$ & $3 \pm 3^{\mathrm{D}}$ \\
Agua $80^{\circ} \mathrm{C}$ & $75 \pm 6^{\mathrm{B}}$ & $35 \pm 9^{\mathrm{C}}$ & $60 \pm 18^{\mathrm{C}}$ & $28 \pm 3^{\mathrm{C}}$ & $0 \pm 0^{\mathrm{D}}$ \\
AS 30 & $100 \pm 0^{\mathrm{A}}$ & $100 \pm 3^{\mathrm{A}}$ & $90 \pm 3^{\mathrm{A}}$ & $93^{\mathrm{A}}$ & $93 \pm 3^{\mathrm{A}}$ \\
AS 50 & $100 \pm 0^{\mathrm{A}}$ & $95 \pm 3^{\mathrm{A}}$ & $85 \pm 10^{\mathrm{A}}$ & $98 \pm 3^{\mathrm{A}}$ & $100 \pm 0^{\mathrm{A}}$ \\
\hline
\end{tabular}

Tabla 6. Tiempo medio de germinación (media \pm error estándar) para cada tratamiento de escarificación y sitio de colecta de semillas de Enterolobium contortisiliquum. Letras diferentes indican diferencias significativas entre las medias de acuerdo a la prueba de comparaciones múltiples de medias DGC.

\begin{tabular}{|c|c|c|c|c|c|}
\hline \multirow{2}{*}{ Tratamiento } & \multicolumn{5}{|c|}{ Poblaciones } \\
\hline & Choromoro & Güemes & H. Irigoyen & Metán & Yuto \\
\hline Testigo & $72,75 \pm 0^{\mathrm{E}}$ & -- & $113 \pm 2^{B}$ & $93,63 \pm 17,38^{D}$ & $90,5 \pm 0^{D}$ \\
\hline Lija & $115,95 \pm 0,36^{A}$ & $112,8 \pm 2,15^{\mathrm{C}}$ & $116,68 \pm 0,32^{A}$ & $114,5 \pm 0,19^{\mathrm{B}}$ & $113,13 \pm 0,69^{B}$ \\
\hline Agua fría & $111 \pm 0^{c}$ & $105,1 \pm 7,32$ в & $53 \pm 0^{\mathrm{F}}$ & $93 \pm 9^{D}$ & $115 \pm 0^{\mathrm{B}}$ \\
\hline Agua $80^{\circ} \mathrm{C}$ & $68,48 \pm 5,85^{\mathrm{E}}$ & $96,98 \pm 4,34^{\mathrm{D}}$ & $67,18 \pm 7,09 \mathrm{E}$ & $78,29 \pm 12,8^{\mathrm{E}}$ & -- \\
\hline Acido S 30' & $114,6 \pm 0,54^{\text {в }}$ & $111,68 \pm 1,38^{c}$ & $115,34 \pm 0,36^{B}$ & $114,64 \pm 0,33^{\text {B }}$ & $110,8 \pm 0,81^{\mathrm{C}}$ \\
\hline Acido S 50' & $115,3 \pm 0,79^{\mathrm{B}}$ & $114,78 \pm 0,22^{\text {B }}$ & $116,15 \pm 0,22^{A}$ & $113,32 \pm 1,12^{B}$ & $109,4 \pm 1,5^{c}$ \\
\hline
\end{tabular}

fueron significativamente mayores para las semillas con los tratamientos de escarificación con lija y con ácido (109,4-116,68). Las velocidades de germinación menores se registraron para el tratamiento con agua a $80{ }^{\circ} \mathrm{C}(68,48-96,98)$ (Tabla 6, Fig. 3).

Según nuestro estudio, no existe variación en la eficiencia de los tratamientos pregerminativos para provocar la salida de la dormición, ya que en todas las poblaciones los mejores métodos fueron la escarificación mecánica y química, logrando tanto un mayor porcentaje de semillas germinadas como una mayor velocidad de germinación. La inmersión en agua caliente $\left(80^{\circ} \mathrm{C}\right)$ seguida del enfriamiento natural por 36 horas, resultó efectiva en semillas de algunas poblaciones, dando valores de proporción de germinación superiores al $50 \%$. Los valores más altos se registraron en una población de condiciones semiáridas (Choromoro) $(0,75)$ y en otra de condiciones subtropicales $(H$. Yrigoyen) $(0,60)$. Si bien se logró relativamente alta germinación, estos valores se alcanzaron hacia el final del experimento (20-30 días desde la siembra), gradualidad mencionada previamente ( $\mathrm{Li}$ et al.,1999; Baskin \& Baskin, 2014; Galíndez et al., 2015). Dada la dificultad de obtener semillas sanas para la propagación de E. contortisiliquum y que los tratamientos con ácido sulfúrico aplicados no afectan la viabilidad de las semillas, se recomienda 
Bol. Soc. Argent. Bot. 51 (1) 2016
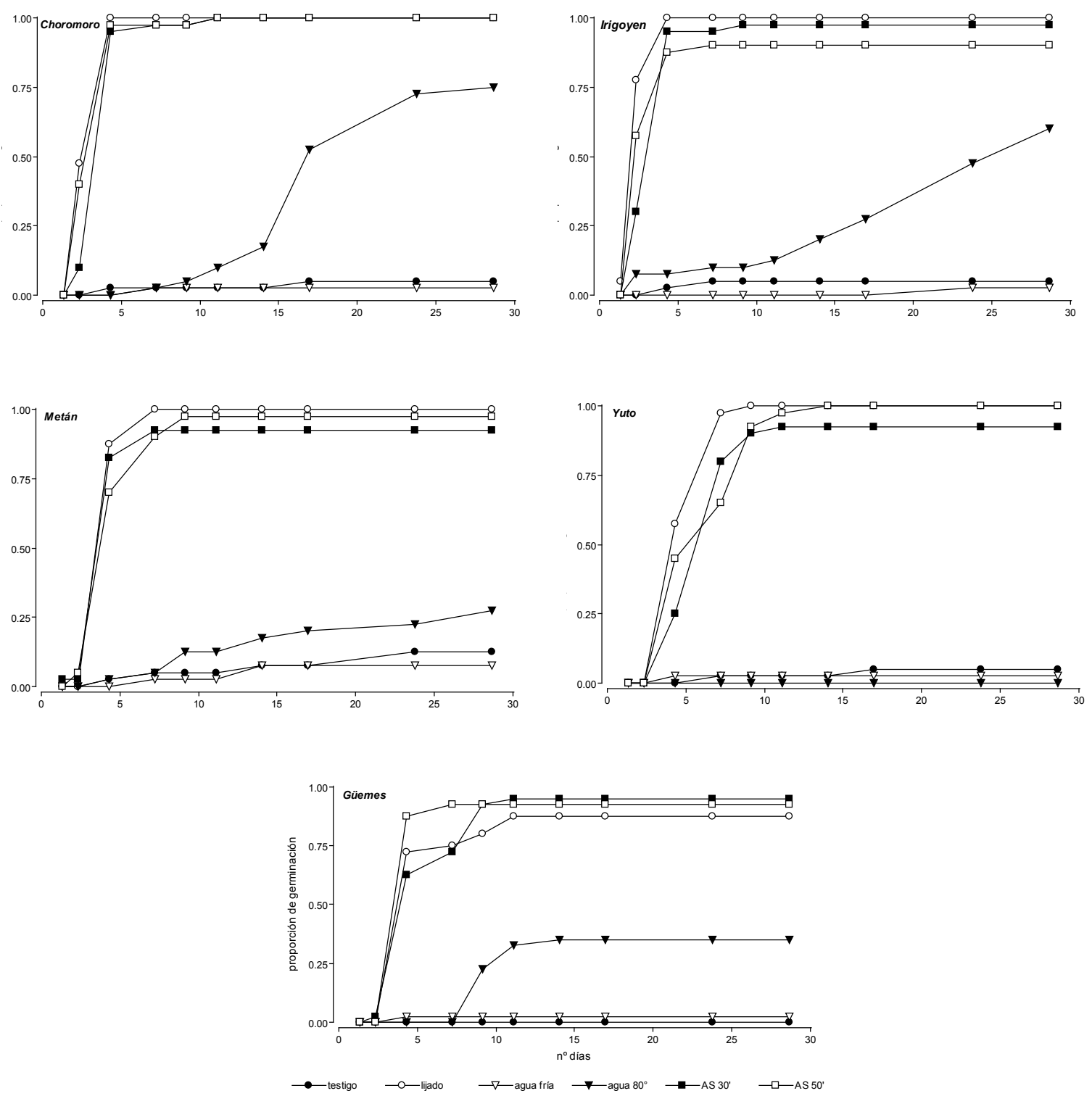

Fig. 3. Velocidad de germinación acumulada para cada población según pretratamientos aplicados.

para las poblaciones de la Selva Pedemontana de Argentina la escarificación con ácido sulfúrico 98 $\%$ durante 30 minutos.

\section{Bibliografia}

ALEXANDRE, R. S., F. G. GONÇALVES, A. P. ROCHA, M. P. DE AMUDA \& E. R. LEMES de Q. 2009. Tratamentos físicos y químicos na superaçâo de dormência em sementes de Enterolobium contortisiliquum (Vell.) Morong. Agrária 4: 156159.

BARTH, S. R., B. I. EIBL \& F. MONTAGNINI. 2008. Adaptabilidad y crecimiento de especies nativas en áreas en recuperación del noroeste de la provincia de Misiones. XIII Jornadas Técnicas Forestales y Ambientales de la Facultad de Ciencias Forestales de la Universidad Nacional de Misiones y EEA Montecarlo, INTA. Eldorado, Misiones. p. 1-16. 


\section{E. C. Lozano et al. - Pretratamientos en semillas de Enterolobium contortisiliquum}

BASKIN, J. M., C. C. BASKIN \& L. XIAOJIE. 2000. Taxonomy, anatomy and evolution of physical dormancy in seeds. Plant Spec. Biol. 15: 139-152.

BASKIN, C. C. \& J. M. BASKIN. 2014. Seeds: Ecology, biogeography and evolution of dormancy and germination. Academic Press, San Diego.

BEWLEY, J. D. \& M. BLACK. 1982. Physiology and biochemistry of seeds II. Springer-Verlag, Berlín.

BIANCHI, A. R. \& C. E. YAÑEZ. 1992. Las precipitaciones en el Noroeste Argentino. Instituto Nacional de Tecnología Agropecuaria, INTA EEA SALTA, Salta.

BORGES, E. E. L., R. C. G. BORGES \& F. F. F. TELES. 1980. Avaliação da maturação e dormência de sementes de orelha-de-negro. Revista Brasil. Sementes 2: 29-32.

CABRERA, A. L. 1994. Regiones Fitogeográficas Argentinas. Enciclopedia argentina de agricultura y jardinería. 2nd ed. ACME, Buenos Aires.

CAPELANES, T. M. C. 1991. Quebra de dormência de sementes florestais em laboratório. Simpósio Brasileiro sobre Sementes Florestais 2: 41. Instituto Forestal, São Paulo.

DI RIENZO, J. A., F. CASANOVES, M. G. BALZARINI, L. GONZALEZ, M. TABLADA \& C. W. ROBLEDO. 2014. InfoStat Versión 2014. Grupo InfoStat, FCA, Universidad Nacional de Córdoba. URL. http//www infostat.com.ar.

EIRA, M. T. S., R. W. FREITAS \& C. M. C. MELLO. 1993. Superação da dormência de sementes de Enterolobium contortisiliquum (Vell.) MorongLeguminosae. Revista Brasil. Sementes 15: 177-181.

GALÍNDEZ, G., G. MALAGRINA, D. CECCATO, T. LEDESMA, L. LINDOW-LÓPEZ \& P. ORTEGABAES. 2015. Dormición física y conservación ex situ en semillas de Amburana cearensis y Myroxylon peruiferum (Fabaceae). Bol. Soc. Argent. Bot. 50: 153-161.

HOC, P. S. 2005. Inga J. F. Mill. En: ANTON, A. M. \& F. O. ZULOAGA (eds.), Flora Fanerogámica Argentina 93: 14-19. IMBIV (CONICET), Programa PROFLORA, Córdoba.

HOPKINS, M. S. \& A. W. GRAHAN.1987. The viability of seeds of rain forest species after experimental soil burial under tropical wet lowland forest in northeastern Australia. Austral Ecol. 12: 97-108.

LEWIS, G. \& L. RICO ARCE. 2005. Tribe Ingeae Benth. En: LEWIS, G., B. SCHRIRE, B. MACKINDER \& M. LOCK (eds.), Legumes of the World, pp. 193210. Royal Botanical Gardens, Kew.
LI, X., J. M. BASKIN \& C. C. BASKIN. 1999. Seeds morphology and physical dormancy of several North American Rhus species (Anacardiaceae). Seed Sci. Res. 9: 247-258.

LIMA, C. M. R., F. BORGHETTI \& M. B. DE SOUSA. 1997. Temperature and germination of the Leguminosae Enterolobium contortisiliquum. Braz. J. Plant Physiol. 9: 97-102.

MALVASI, U. C. \& M. MATTOS MALVASI. 2004. Dormancy breaking and germination of Enterolobium contortisiliquum (Vell.) Morong seed. Arq. Biol. Technol. 47: 1-4.

MORANDINI, M. N. \& M. I. DE VIANA.2009. Depredación pre-dispersiva de semillas en tres poblaciones del árbol Enterolobium contortisiliquum (Fabaceae). Revista Biol. Trop. 57: 1-7.

NAVARRO, M., A. MESA \& Y. GONZÁLEZ. 2002. Capacidad germinativa de las semillas de Albizia lebbeck (L.) Benth. II. Ruptura de dormancia y emergencia de plántulas. Pastos y Forrajes 25: 202210.

PRITCHARD, H. W. \& A. P. MILLER. 1995. The effects of constant temperatures, light and seed quality on the germination characteristics of Agave americana. Bol. Soc. Bot. México 57:11-14.

RAMÍREZ, M., B. CARBALLO, A. URDANETA \& D. E. GARCÍA. 2013. Emergencia y desarrollo inicial de cuatro leguminosas forrajeras arbóreas presentes en la altiplanicie de Maracaibo, Venezuela. Pastos y forrajes 36: 303-312.

ROSSINI OLIVA, S., M. C. VALDÉS, F. ANDRÉS, F. MÁRQUEZ CAMPÓN \& M. BUESO LÓPEZ. 2006. Germinación de las semillas en algunas especies americanas de Fabaceae y Bignoniaceae cultivadas en Sevilla (50 especies). Lagascalia 26: 119-129.

TORTORELLI, L. A. 2009. Maderas y Bosques Argentinos I. Orientación Gráfica Editora, Buenos Aires.

Recibido el 2 de julio de 2015, aceptado el 11 de diciembre de 2015. 
